\title{
Redes e divulgação do Unbral Fronteiras
}

\author{
Adriana Dorfman' \\ Karla Maria Müller"
}

\section{RESUMO}

Um dos propósitos do projeto Unbral Fronteiras - Portal de Acesso Aberto das Universidades Brasileiras sobre Limites e Fronteiras é consolidar o campo dos Estudos Fronteiriços. Nessa tarefa convergem várias atividades, como a cooperação com universidades, a participação em redes de pesquisadores, em associações, em grupos de pesquisa, a frequência a eventos, a oferta de cursos, a publicação de textos sobre fronteiras e a divulgação dessas instâncias. 0 artigo aborda a assinatura de termo entre a UNIPAMPA e a UFRGS. Instâncias internacionais relevantes como a Association for Borderland Studies e o congresso Border Regions in Transition, entre outras redes internacionais dedicadas aos Estudos Fronteiriços, são apresentadas. Na região destacam-se os eventos Seminário de Estudos Fronteiriços e Geofronteras. Entre redes de pesquisadores/ grupos de pesquisa, são relevantes o Grupo Retis e o AntiAtlas das Fronteiras, entre tantos mais. Os pesquisadores envolvidos na produção do Portal Unbral das Fronteiras Brasileiras reúnem-se no GREFIT - Grupo de Pesquisa Espaço, Fronteira, Informação, Tecnologia.

PALAVRAS-CHAVE: Estudos Fronteiriços, Cooperação Universitária, Association for Borderland Studies, Seminário de Estudos Fronteiriços, Grupo Retis, GREFIT

I Professora do Departamento de Geografia e do Programa de Pós-Graduação em Geografia da Universidade Federal do Rio Grande do Sul. Coordenadora do projeto Unbral Fronteiras - Portal deAcesso Aberto das Universidades Brasileiras sobre Limites e Fronteiras. Líder do GREFIT - Grupo de Pesquisas Espaço Fronteira Informação Tecnologia. E-mail: adriana.dorfman@ufrgs.br

II Professora do Programa de Pós-Graduação em Comunicação e Informação da Universidade Federal do Rio Grande do Sul; Vice-coordenadora da pesquisa do Unbral Fronteiras - Portal de Acesso Aberto das Universidades Brasileiras sobre Fronteiras e Limites. E-mail: kmmuller@ufrgs.br 


\section{APRESENTAÇÃO}

Um dos propósitos do projeto Unbral Fronteiras - Portal de Acesso Aberto das Universidades Brasileiras sobre Limites e Fronteiras é consolidar o campo dos Estudos Fronteiriços. Nessa tarefa convergem várias atividades, como a cooperação com universidades, a participação em redes de pesquisadores, em associações, em grupos de pesquisa, a frequência a eventos, a oferta de cursos, a publicação de textos sobre fronteiras e a divulgação dessas instâncias. A seguir, listamos atividades e instituições em que a equipe do Unbral Fronteiras participou em 2015.

\section{OS TERMOS DE COOPERAÇÃO TÉCNICA}

O projeto previa originalmente a assinatura de termos de cooperação entre as universidades parceiras. No ano de 2015, revimos 0 alcance de tais termos no que tange a direitos autorais: as universidades têm direito moral e patrimonial sobre os trabalhos produzidos pelos pesquisadores nelas atuando? A pergunta não possui resposta única.

Além disso, a coleção construída em 2015 se voltou para teses e dissertações publicadas em repositórios institucionais, de acesso aberto. Nessas bases de dados, a instituição se compromete com a manutenção, preservação e acesso. Assim, não há necessidade de permissões especiais para análise, tendo-se o cuidado de manter as referências ao site de publicação original.

Um termo de cooperação científica foi assinado entre a UFRGS e a UNIPAMPA. As monografias da UNIPAMPA não estão disponíveis integralmente em um repositório institucional e o termo tem sido útil para permitir a organização da coleção de monografias defendidas nesta Universidade. 


\title{
REDES E ASSOCIAÇÕES
}

Os Estudos Fronteiriços, talvez por suas características multidisciplinares, motivam muitas iniciativas para circulação de informação. Pedimos desculpas aos grupos e redes que não constam da lista que segue, esperando seu contato para inclusão.

Entre as associações dedicadas aos Estudos Fronteiriços, a mais eminente é a Association for Borderland Studies (ABS). Além de encontros anuais, foi realizado um encontro mundial em 2014 (Joensuu/FI-São Petesburgo/RU) e outro está previsto para 2018. Através dessa Associação, temos circulado informação sobre os eventos científicos organizados no Brasil para a comunidade internacional. Há uma proposta de construir um capítulo regional na América do Sul dessa Associação.

A página da Association for Borderland Studies informa, em inglês e em espanhol, que:

\begin{abstract}
A Associação dos Estudos Fronteiriços (ABS) é a principal entidade internacional e acadêmica a se dedicar exclusivamente ao intercâmbio constante de ideias e informações relacionadas com as áreas fronteiriças internacionais. Fundada em 1976, originalmente com ênfase nos Estudos Fronteiriços entre 0 México e os Estados Unidos, a associação tem tido constante crescimento. Atualmente, essa sociedade interdisciplinar de membros acadêmicos agrupa mais de cem instituições governamentais e acadêmicas e ONGs presentes na América, Ásia, África e Europa.
\end{abstract}

O endereço da ABS é <http://absborderlands.org/about/enespanol/>

Outra rede muito relevante nos Estudos Fronteiriços internacionais é chamada Border Regions in Transition (BRIT), ou Regiões Fronteiriças em Transição. Essa rede se organiza em torno de conferências bienais. A página da edição 2016 da 
conferência BRIT informa que esse encontro científico internacional e interdisciplinar sobre fronteiras e limites tem se realizado desde 1994 (<http://www.sdu.dk/en/om_sdu/institutter_centre/i_graenseforskning/ events/brit+2016/about+brit2016>).

Cabe ainda citar a Comissão de Geografia Política da União Geográfica Internacional (CGP-UGI), que se dedica aos Estudos Fronteiriços em concomitância com outros campos da Geografia Política. A Comissão de Geografia Política integra a União Geográfica Internacional (UGI), uma organização profissional dedicada ao desenvolvimento da disciplina geográfica. A UGI organiza um congresso a cada quatro anos e promove conferências regionais anualmente. Vejamos, por exemplo, a frase de abertura, em inglês, do site da CGP: "Com o fim do período dos "finalismos" [endisms], todos concordamos que o mundo não é, de modo algum, sem fronteiras. As fronteiras estão aí, mais "concretas" do que nunca, uma vez que muitas delas se transformaram em novos muros" (<www.igu-cpg.unimib.it>).

Da mesma forma, muitos são os grupos no Facebook que se dedicam a divulgação de iniciativas ligadas às fronteiras. A seguir, listamos alguns grupos e páginas brasileiros e internacionais.

O Grupo Retis é uma "rede de pesquisadores dedicada à pesquisa de limites e fronteiras internacionais. Atua no Departamento de Geografia da UFRJ, com apoio do CNPq e a participação de pesquisadores associados de outras instituições", conforme consta em <https://www. facebook.com/GrupoRetis/>

O Observatório da Fronteira é "uma plataforma que reúne notícias e análises da conjuntura política, econômica e social da região fronteiriça brasileira". Visite a página em: <https://www.facebook.com/ observatoriodafronteira/?fref=ts>

O grupo Fronteiras Culturais/Fronteras Culturales trata de "processos de integração entre os povos, promovidos por coletivos culturais autônomos, artistas, produtores(as) e pesquisadores(as)", como consta em <https://www.facebook.com/Fronteiras-Culturais-FronterasCulturales-971969389535396/?fref=ts> 
Fronteras Latinoamericanas é "uma plataforma que reúne notícias e análises da conjuntura política, econômica e social da região fronteiriça" e está disponível em <https://www.facebook.com/ groups/416832461801455/?fref=ts>

Entre as páginas que não são publicadas na América do Sul, mas que também são relevantes, conhecemos Border Aesthetics. Esta página em inglês e em outras línguas se propõe a ser "um espaço no Facebook para discussão e informação sobre estética fronteiriça, iniciado pelo projeto de pesquisa transdisciplinar "Border Aesthetics" (...) e pelo Grupo de Pesquisa "Poéticas da Fronteira", da Universidade de Tromsø", na Noruega, conforme a página <https://www.facebook.com/ groups/144219115609995/>.

Por fim, o Antiatlas of Borders traz posts de línguas e origens dispersas pelo mundo, e se apresenta como "um programa exploratório e transdisciplinar que relata as mutações das fronteiras do século XXI através de pesquisa, arte e prática". Muito mais pode ser conhecido em: <https://www.facebook.com/antiAtlasdesfrontieres/>.

\section{GRUPOS DE PESQUISA}

Os grupos abaixo descritos contam com membros da equipe do Unbral Fronteiras. Uma lista completa de grupos de pesquisa dedicada ao estudo de fronteiras, elaborada a partir do Diretório de Grupos de Pesquisa do CNPq, pode ser encontrada no Apêndice I.

\section{Grupo de Pesquisa Espaço, Fronteira, Informação e Tecnologia - GREFIT \\ Objetivos}

Os pesquisadores, estudantes e técnicos desse grupo buscam explorar os aspectos ontológicos e epistemológicos de fronteiras, territórios e espaços geográficos diante da difusão de tecnologias de informação. Sua experiência em Estudos 
Fronteiriços e nas práticas necessárias para o tratamento com informação digital e desenvolvimento web foca-se no acesso aberto ao conhecimento. $O$ trabalho do grupo possui inserção internacional, bem como expressão nacional estabelecida, colaborando com a descrição e análise de processos territoriais e seus condicionantes informacionais e técnicos. O Portal de Acesso Aberto das Universidades Brasileiras sobre Limites e Fronteiras - Unbral Fronteiras, integrando diferentes IES brasileiras, é uma de suas materializações.

\section{Linhas de pesquisa}

\section{Análise dos fluxos transfronteiriços (i)legais no Brasil e em diferentes escalas.}

Estudos Fronteiriços de cunho qualitativo e quantitativo das práticas legais e ilegais em seus aspectos territoriais. Estabelecimento de metodologias de pesquisa sobre 0 contrabando; levantamento de dados sobre as diferentes práticas, redes e percursos associados a distintos tipos de contrabando. Discussão sobre a produção de normas, sua aplicação e suas relações com territórios, espaços e lugares. Descontinuidades espaciais.

\section{Informação digital e desenvolvimento web.}

Estudos de cunho teórico e aplicado, abordando a produção, comunicação e o uso de informação digital para sistemas de gerenciamento de conteúdo e de documentos web do tipo open source (CMS, LMS, Repositórios).

\section{Endereço}

<https://dgp.cnpq.br/dgp/espelhogrupo/5812863341122235> 


\section{Integrações Econômicas Binacionais e Desenvolvimento Social em Regiões de Fronteiras (GEIEB) \\ Objetivos}

Investigar a integração econômica entre o Brasil e o Uruguai e 0 desenvolvimento social em regiões de fronteiras nos dois países. Estudar acordos, tratados e outros documentos celebrados no campo econômico; Estudar acordos, tratados e outros documentos celebrados sobre regiões de fronteiras; Paradiplomacia: verificar como instituições fora do âmbito do Estado, organizam-se e propõem soluções de problemas em regiões de fronteiras; Verificar o Comércio Exterior entre os dois países e em especial entre as cidades gêmeas; Desenvolvimento Social em Regiões de Fronteiras. Turismo fronteiriço como fator de desenvolvimento econômico.

\section{Linhas de pesquisa}

Acordos e Tratados entre o Brasil e o Uruguai;

Comércio Exterior e Cidades-Gêmeas;

Desenvolvimento em Regiões de Fronteiras;

Integração Econômica Binacional;

Organizações privadas fora do âmbito do Estado e desenvolvimento fronteiriço;

Turismo e integração regional.

\section{Endereço:}

<https://dgp.cnpq.br/dgp/espelhogrupo/4564857139824219>

\section{Grupo Retis}

\section{Objetivos}

A pesquisa sobre redes de tráfico de drogas ilícitas e lavagem de dinheiro teve forte repercussão no meio governamental e na mídia. O uso dos mapas e dos textos produzidos foi de ajuda 
para a Receita Federal, a Secretaría Anti-drogas, a UNESCO, a UNODC-Brasil e a UNODC-Viena. Também resultou na Cátedra UNESCO sobre Drogas llícitas. A metodologia desenvolvida foi aplicada em outras áreas do Brasil por órgãos federais. A pesquisa sobre a Faixa de Fronteira Continental do Brasil e interações com a América do Sul fundamentou a elaboração do Programa de Desenvolvimento da Faixa de Fronteira do Ministério da Integração, que serve de base para a atual Comissão Permanente para o Desenvolvimento e a Integração da Faixa de Fronteira. Os projetos dos editais universais do CNPq e o Projeto PROSUL/CNPq propiciaram ampliar o estudo para outras fronteiras internacionais sul-americanas. Recentemente, o grupo realizou diagnóstico sobre segurança pública na Faixa de Fronteira para a ENAFRON/SENASP/MJ.

\section{Linhas de pesquisa}

Conservação em Zona de Fronteira;

Geografia das Drogas llícitas;

Sistema Bancário-Financeiro e a Geopolítica da Segurança; Limites e Fronteiras Internacionais na América do Sul;

Pensamento Geográfico e Organização do Território;

Redes, Território e Governo Local na Amazônia.

\section{Endereço}

<https://dgp.cnpq.br/dgp/espelhogrupo/5548233559891298>

\section{Laboratório de Estudos e Pesquisas Internacionais e de Fronteira}

Objetivos

O Lepif foi criado para o desenvolvimento de pesquisas e ensino sobre as fronteiras em sentido amplo, envolvendo estudos e projetos com temáticas internacionais, interculturais e multidiciplinares. O Laboratório visa proporcionar um espaço 
para a produção de atividades relacionadas às diversas áreas das ciências sociais e humanas, buscando ampliar a produção acadêmica sobre as fronteiras de forma colaborativa entre Instituições de Ensino e de Pesquisa, Alunos, Pesquisadores e Gestores Públicos.

\section{Linhas de pesquisa}

O Lepif faz parte da Rede de Pesquisa Fronteras Globales, formada por dez países da América Latina e a Itália, a qual desenvolve o Projeto "Explorando la economía política de la violencia en los sistemas fronterizos de América Latina: hacía una comprensíon integral" coordenada pela Facultad Latinoamericana de Ciencias Sociales - FLACSO Equador e financiada pelo International Development Research Centre - IDRC Canadá. A equipe do Laboratório participou da pesquisa de campo do Projeto "Percepção social sobre segurança e política de drogas na zona de fronteira" do Instituto de Pesquisa Econômica Aplicada - IPEA e executado pela empresa de consultoria especializada Consulting do Brasil. Direito, Fronteiras e Relações Internacionais: Esta linha de pesquisa visa promover estudos empíricos e teóricos na temática do Direito e suas interfaces com as Relações Internacionais e a temática das Fronteiras. A proposta é desenvolver pesquisas compreendendo as fronteiras de forma ampla, como as existentes entre as diferentes áreas do conhecimento, culturas, etnias etc. $\mathrm{O}$ grupo de pesquisadores é formado por estudantes do curso de Direito e de Relações Internacionais, e está aberto à toda forma de diálogo e colaboração acadêmica possível.

\section{Endereço}

$<$ <https://www.lepif.org/> 


\section{EVENTOS}

A equipe do Unbral Fronteiras se fez presente nos seguintes eventos de pesquisa, fazendo a divulgação do projeto:

- Participação no Encuentro Internacional de Investigadores "Tejiendo Redes", promovido pelo Escritório das Nações Unidas contra a Droga e o Crime na Colômbia - UNODC, em Bogotá, Colômbia, entre 5 e 6 de março de 2015:

Apresentação do trabalho: "Morals, norms and infrastructure as keys to geographical study of smuggling and its repression at Brazilian borders" (Moral, norma e infraestrutura como chaves para a análise geográfica do contrabando e de sua repressão nas fronteiras brasileiras), de autoria de Adriana Dorfman.

- Participação no Association for Borderland Studies/ABS Annual Meeting 2015 (Encontro Anual da Associação de Estudos Fronteiriços 2015) em Portland, EUA, entre 08 e 11 de abril de 2015:

Apresentação do trabalho "Geografias morales del contrabando", de autoria de Adriana Dorfman

- Participação no V SEF, Seminário de Estudos Fronteiriços, promovido pelo Laboratório de Estudos Fronteiriços - Programa de Pós-Graduação em Estudos Fronteiriços - UFMS, Campus Pantanal da Universidade Federal de Mato Grosso do Sul, entre 20 e 22 de maio de 2015:

Apresentação do trabalho "Fraturas e fronteiras no espaço urbano da educação", de autoria de Lizandra Vega da Cunha e Adriana Dorfman;

Lançamento do Anuário do Unbral das Fronteiras Brasileiras 2014; 
Realização da Mesa redonda: Grupos de Estudos Fronteiriços e Portal Unbral Fronteiras

- Participação na oficina UPMS: Mulheres em Diálogo de Fronteiras, organizada pela Universidade Popular dos Movimentos Sociais, através do coletivo Cotidiano Mujer, Centro Universitario de Rivera (UDELAR) e do Inmujeres do Ministerio de Desarollo Social/MIDES(Uruguai), e dos Departamentos de Sociologia e Geografia da Universidade Federal do Rio Grande do Sul (UFRGS), da Coordenadoria da Mulher da Prefeitura de Sant'Ana do Livramento (Brasil), em Rivera-Sant'Ana do Livramento, entre 6 e 8 de agosto de 2015:

Coordenação do eixo "Autonomia Territorial".

- Participação na International Geographic Union Regional Conference Moscow 2015 (Conferência Regional da União Geográfica Internacional 2015), em Moscou, Rússia, entre 17 e 21 de agosto de 2015:

Participação na reunião da Comissão de Geografia Política da UGl;

Participação na reunião dos delegados latino-americanos com a diretoria da UGI;

Coordenação da sessão "Borders and Illegalities" (Fronteiras e llegalidades), por Adriana Dorfman e Elena dell'Agnese;

Apresentação do trabalho: "Securitization and Stigma at the Peace Border" (Securitização e estigma na Fronteira da Paz), de autoria de Adriana Dorfman e Arthur Borba Colen França.

- Participação no III Seminário Internacional dos Espaços de Fronteira (III Geofronteras): Integração, Cooperação e Conflitos, no campus da Universidade Nacional de Itapúa, Encarnación, Paraguai, entre 08 e 10 de setembro de 2015. 
- Participação no XI ENANPEGE, Encontro Nacional da ANPEGE promovido pela Associação Nacional de PósGraduação em Geografia, em Presidente Prudente, SP, entre 9 e 12 de outubro de 2015:

Coordenação do Grupo de Trabalho "Estado, Território e Fronteira".

Lançamento do Anuário Unbral das Fronteiras Brasileiras 2014.

- Participação na conferência Castle-talks on cross-border cooperation (Conversações no Castelo sobre Cooperação Transfronteiriça), em Estrasburgo, França, entre 07 e 10 de dezembro de 2015:

Discussão do tema: "Borders: source of conflict or place of reconciliation?" (A fronteira: fonte de conflito ou lugar de reconciliação?)

Apresentação do trabalho: "Smugglers and flawed citizenship" (Contrabandistas e cidadania falhada), de autoria de Adriana Dorfman.

\section{CURSOS MINISTRADOS}

Entre as atividades de promoção do projeto e dos Estudos Fronteiriços, foi ministrado um curso de pós-graduação no Programa de Pós-Graduação em Geografia da UFRGS. Tratase do seminário concentrado "Estudos Fronteiriços: fronteiras e migrações em perspectiva descolonial", ministrado pelos professores Adriana Dorfman (UFRGS) e Daniel Angel Burgueño Etcheverry (UNIPAMPA).

Este seminário foi realizado em uma perspectiva binacional, multiescalar e interdisciplinar sobre os espaços fronteiriços. Para encaminhar conceitual e empiricamente seu entendimento, se fez necessário realizar um breve percurso pelas origens dos limites 
interestatais e pelos estudos geopolíticos clássicos e nacionais, que moldaram o objeto geográfico e as formas de pensar e trabalhar com categorias tais como limite e fronteira. Outros conceitos relevantes como frentes, barreiras e fraturas, foram também tematizados, ampliando a discussão a outros objetos, além dos limites internacionais.

O objetivo geral do seminário foi:

Discutir as fronteiras. Discutir as migrações internacionais. Apresentar a formação dos limites territoriais do estado. Revisar as abordagens sobre os conceitos de território, limite e fronteira a partir dos estudos clássicos no Brasil. Relacionar as abordagens em distintos campos disciplinares com formas e processos contemporâneos (frentes de expansão, barreiras comerciais e fronteiras tecnificadas, fraturas urbanas). Abordar a contemporaneidade das fronteiras a partir das experiências migratórias e das teorias descoloniais. Orientar os discentes para a realização de suas investigações a partir da discussão de seus projetos.

Os objetivos específicos do seminário foram:

Apresentar as principais constelações conceituais empregadas nos Estudos Fronteiriços; discutir as relações disciplinares em jogo; propor uma operacionalização dos conceitos de limites e fronteiras, barreiras, frentes e fraturas. Relacionar limites, fronteiras, descontinuidades e desigualdades; formação territorial e delimitação; fronteiras e configuração de redes multiescalares; fronteiras e mobilidades; fronteiras e territorialidades multiescalares.

Apresentar os principais aportes em circulação no estudo das migrações e mobilidades. Discutir documentação e outros recursos de controle das populações. Discutir estudos de caso.

Revisar e discutir as abordagens teórico-metodológicas destas categorias a partir das Ciências Humanas no Brasil; estudar a atualidade das fronteiras políticas à luz do discutido. 
Debater alcances e limites das metodologias e técnicas de pesquisa em uso; compartilhar experiências de pesquisa em campo; estimular a sensibilidade ética na realização das pesquisas.

\section{TEXTOS PUBLICADOS}

\section{Livros organizados}

DORFMAN, Adriana (Coord.). Anuário Unbral das fronteiras brasileiras 2014. Porto Alegre: Editora Letra1, 2015. 130 p. Disponível em <http://www.ufrgs.br/ igeo/ig/arquivo/Anuario_Unbral_WEB.pdf>.

DORFMAN, Adriana et al. Territórios e lugares da Região Metropolitana de Porto Alegre. Porto Alegre: Editora Letra1, 2015. 155 p. Disponível em <https:// issuu.com/editoraletra1/docs/cartilha_territorios_e_lugares_rmpa>.

RADDATZ, Vera R. S.; MÜLLER, Karla M. (Orgs.). Comunicação, cultura e fronteiras. ljuí, Ed. Unijuí, 2015.

\section{Capítulo de livro}

DORFMAN, Adriana. Enafron e suas materializações no Rio Grande do Sul . In: MALLMANN, M. Isabel (org.) Fronteiras e relações Brasil-Uruguai. p. 199-212. Disponível em <http://ebooks. pucrs.br/edipucrs/Ebooks/Pdf/978-85-397-0691-4.pdf>.

DORFMAN, Adriana. Geography of cultural imagination: constructing and representing the border between Brazil and Uruguay. In: Centre for Advanced Study Sofia. LILOVA, Dessislava (Org.). Natural Sciences, Technologies, and Social Worlds. 1ed. Sofia: Centre for Advanced Study Sofia, 2015, v. 1, p. 101-129. (em búlgaro).

DORFMAN, Adriana. Smuggling : power networks, moral geographies and norm enforecement at work at Southern Cone borders. In: AMILHAT SZARY, Anne-Laure; GIRAUT, Fréderic (orgs.).Borderities and the politics of contemporary mobile border, New York: Palgrave/Macmillan, 2015, p. 171-187, il. (em inglês)

DORFMAN, Adriana; ASSUMPÇÃO, Marla Barbosa. Brazil-Uruguay : Brazilian Island, Masoller and Mirim Lagoon. In: BRUNET-JAILLY, Emmanuel (ed.). Border disputes: a global encyclopedia., Santa Barbara, California: ABC-CLIO, 2015, p. 471-482. (em inglês)

DORFMAN, Adriana; FRANÇA, Arthur Borba Colen; ASSUMPÇÃO, Marla Barbosa. State borders in South America . In: Introduction to Border Studies, Vladivostok: Dalnauka, 2015, p. 265-283. (em inglês)

MÜLLER, Karla M. Mídia local fronteiriça: do impresso ao online. In: RADDATZ, Vera R. S.; MÜLLER, Karla M.(orgs.). Comunicação, cultura e fronteiras. ljuí, Ed. Unijuí, 2015, p. 117-137. 


\section{Anais de evento nacional}

MÜLLER, Karla M. BARTHS, Camila; COSTA, Stefânia. Interfaces da cultura organizacional no contexto fronteiriço: possibilidades, estratégias e governança corporativa. In: Anais Congresso Brasileiro Científico de Comunicação Organizacional e de Relações Públicas. (9. : 2015, maio : Campinas, SP) [recurso eletrônico]. Porto Alegre, RS : ediPUCRS, 2015, p. 418-433.

\section{Artigo em periódico científico}

DORFMAN, Adriana. Contrabando: pasar es la respuesta a la existencia de una frontera, burlar es el acto simétrico al control. Aldea mundo: revista sobre fronteras e integración Vol. 20, n. 39 (ene./jun. 2015), p. 33-44 , il. Disponível em <http://www.redalyc.org/ articulo. oa?id=54343963004>. (em espanhol)

DORFMAN, Adriana et al. Redes de Poder e estratégias espaciais dos fiscais municipais em Santana do Livramento (RS) . In: Geographia, Rio de Janeiro : UFF, 2013 Vol. 17, n. 33 (2015), p. 98-125, il. Disponível em <http://www.uff.br/geographia/ojs/index. php/geographia/article/viewArticle/490>.

MÜLLER, Karla M.; RADDATZ, Vera L.; STRASSBURGER, Tabita. LinkMídia e fronteiras: primeiras discussões sobre a cartografia desses estudos no Brasil. Revista Intexto: Edição Especial 20 anos do PPGCOM. № 34. [recurso eletrônico] Porto Alegre: PPGCOM/UFRGS, set-dez/2015, p. 385-400.

\section{Relatório técnico e de pesquisa}

NEVES, Alex Jorge das et al. Segurança pública nas fronteiras: relatório síntese. Brasília, DF: Ministério da Justiça, 2015. 97 p., il.

\section{Tradução}

ROSIÉRE, Stéphane et al. Mundialização e teicopolíticas : análise do fechamento contemporâneo das fronteiras internacionais. In: Boletim Gaúcho de Geografia. Porto Alegre, RS, v. 42, n. 2 (maio 2015), p. 369-388, il. Disponível em <http://seer. ufrgs.br/index.php/bgg/article/view/56327>.

SZARY, Anne Laure Amilhat et al. Artista passa-paredes? . In: Boletim Gaúcho de Geografia. Porto Alegre, RS V. 42, n. 2 (maio 2015), p. 412-434, il. Disponível em <http://www.seer.ufrgs.br/bgg/article/view/56326>. 
